\title{
New neighbours: IV. 30 DENIS late-M dwarfs between 15 and 30 parsecs
}

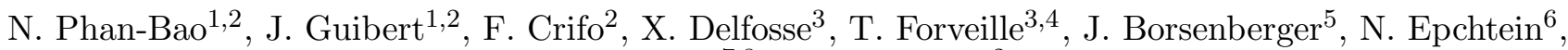 \\ P. Fouqué ${ }^{7,8}$, and G. Simon ${ }^{2}$ \\ 1 Centre d'Analyse des Images, DASGAL, Observatoire de Paris, 61 avenue de l'Observatoire, 75014 Paris, France \\ 2 Observatoire de Paris (DASGAL/UMR-8633), 75014 Paris, France \\ 3 Laboratoire d'Astrophysique de Grenoble, Université J. Fourier, BP 53, 38041 Grenoble, France \\ 4 Canada-France-Hawaii Telescope Corporation, 65-1238 Mamalahoa Highway, Kamuela, HI 96743, USA \\ ${ }^{5}$ Institut d'Astrophysique de Paris, 98bis boulevard Arago, 75014 Paris \\ 6 Observatoire de la Côte d'Azur, Département Fresnel, BP 4229, 06304 Nice Cedex 4, France \\ 7 DESPA, Observatoire de Paris, 5 place J. Janssen, 92195 Meudon Cedex, France \\ 8 European Southern Observatory, Casilla 19001, Santiago 19, Chile
}

Received 16 July 2001 / Accepted 15 October 2001

\begin{abstract}
We present 30 new nearby $(d<30$ pc) red dwarf candidates, with estimated spectral types M 6 to M 8 . 26 were directly selected from the DENIS database, and another 4 were first extracted from the LHS catalogue and cross-identified with a DENIS counterpart. Their proper motions were measured on the MAMA measuring machine from plates spanning 13 to 48 years, and are larger than $0.1 \mathrm{yr}^{-1}$, ruling out the possibility that they are giants. Their distances were estimated from the DENIS colours and IR colour-magnitude relations and range between 15 and 30 pc.
\end{abstract}

Key words. astrometry - proper motions - low mass stars

\section{Introduction}

Much of our understanding of stellar astronomy rests upon the nearest stars. As individual objects they are the brightest and hence best studied examples of their spectral type, and their trigonometric parallaxes can be measured accurately, although with significant effort. The solar neighbourhood sample also provides deep insight into the nature of our Galaxy's components, through studies of its stellar luminosity and mass functions, its kinematics, chemical composition, and multiplicity statistics.

Perhaps surprisingly, this sample is still incomplete, even very close to the Sun, as illustrated by the recent discoveries of three new stars with $d<6$ pc (Henry et al. 1997; Delfosse et al. 2001; Scholz et al. 2001). From a comparison of the observed star densities within 5 and 10 parsecs, Henry et al. (1997) estimate that approximately 130 systems are missing from the $10 \mathrm{pc}$ sample. Most of these missing stars are red $\mathrm{M}$ dwarfs, with $B-V>1.70$ (Gliese et al. 1986), and the deficit is largest south of declination $-30^{\circ}$.

Send offprint requests to: N. Phan-Bao, e-mail: bn.phan@obspm.fr
For the most part the known members of the solar neighbourhood have been selected within the available catalogues of trigonometric parallaxes, though some significant fraction is still included on the basis of photometric or spectroscopic distances (Gliese 1956; Gliese \& Jahreiß 1979, 1991). However, for at least the last fifty years, parallax programs have selected their targets for a good part from proper motion catalogues, such as the Cincinnati catalogues (Porter et al. 1918, 1930), the Luyten catalogues (LFT 1955; LTT 1957, 1961; LHS 1979; NLTT 1980) or the Lowell catalogues (Giclas et al. 1971, 1978). This leads to a proper motion bias of the resulting sample, at least for the faint end. To date even the smaller LHS catalogue has been incompletely followed-up, and the Luyten catalogues therefore contain many unrecognized nearby stars (e.g. Scholz et al. 2001, for an extreme example). Several groups are working to identify them (Henry et al. 1997; Gizis \& Reid 1997; Jahreiß et al. 2001). One limitation to these efforts is the much brighter limiting magnitude of the proper motion catalogues south of declination $-33^{\circ}$ (e.g. Scholz et al. 2001, for a recent discussion), where a much larger fraction of the solar neighbourhood stars is currently missing. 
Table 1. Typical detection limiting distances $\left(D_{\lim }\right)$ for $\mathrm{M}$ dwarfs with colour $2.0 \leq I-J \leq 3.0$ in the DENIS survey. The spectral types are from Leggett (1992) and the absolute magnitude is taken from Fig. 1.

\begin{tabular}{llll}
\hline \hline$I-J$ & Spectral type & $M_{I}$ & $D_{\lim }$ \\
\hline 2.0 & M 5.5-M 6 & 11.5 & $250 \mathrm{pc}$ \\
2.5 & M 6.5-M 7 & 12.9 & $130 \mathrm{pc}$ \\
3.0 & M 8 & 14.0 & $80 \mathrm{pc}$ \\
\hline
\end{tabular}

The recent near-infrared sky surveys, DENIS and 2MASS, represent a powerful alternative tool to identify these faint and cool nearby stars. Candidates are first selected on simple colour and photometric distance criteria, producing an initial list that also contains many giants, and potentially some pre-main-sequence stars. At low galactic latitudes it would also include many reddened distant stars. In a second step these contaminating populations need to be rejected, through either (1) a systematic spectroscopic follow up (Gizis et al. 2000), or (2) proper motion selection (distant giants have very small proper motions), or (3) more accurate multi-colour photometry than available from the surveys. Systematic spectroscopy is clearly the cleanest approach, and does not reject the few nearby star that, like Gl 710 for instance, have small proper motions. Proper motion selection on the other hand is cheap and effective, but it obviously does nothing to correct for the proper motion bias of the present nearby star catalogues.

We are using here the DENIS near infrared sky survey to identify possible very-low-mass stars within 30 parsecs. We conservatively adopt a distance cutoff slightly larger than the 25 pc limit of the Catalogue of Nearby Stars of Gliese and Jahreiß (CNS3, 1991), to avoid losing bona-fide $d<25$ pc stars from imprecise photometric distances. One initial result of this effort was the discovery of an M 9 dwarf at a spectroscopic distance of only 4 pc, DENISP J104814.7-395606.1 (Delfosse et al. 2001). In this paper we present 26 nearby red dwarf candidates, selected with $I-J>2.0$ (M6 or later) in the DENIS database and with a proper motion larger than $0.1 \mathrm{yr}^{-1} .13$ of these are new, and another 13 were known as high-PM objects but had not been characterized further. Four additional candidates were first selected from the southern part of the LHS catalogue and then identified with DENIS counterparts.

Section 2 reviews the sample selection, and Sect. 3 presents the photographic photometry and the proper motion measurements. We discuss the results in Sect. 4 and summarize in Sect. 5.

\section{Sample selection}

\subsection{DENIS observations}

The DEep Near-Infrared Survey (DENIS) is a southern sky survey (Epchtein 1997), which will provide full coverage of the southern hemisphere in two near-infrared bands

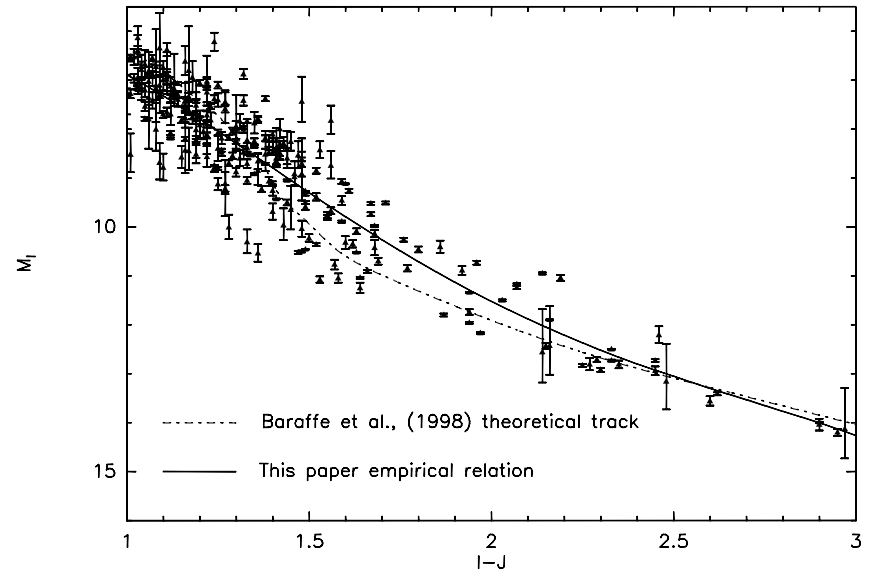

Fig. 1. $M_{I}: I-J$ HR diagram for M dwarfs with known distances (from Leggett 1992 and Tinney et al. 1995). Our empirical polynomial fit and the 3 Gyr model of Baraffe et al. (1998) for a solar metalicity are overlaid.

( $J$ and $K_{\mathrm{S}}$ ) and one optical band (I). DENIS observations are carried out on the ESO $1 \mathrm{~m}$ telescope at La Silla. Dichroic beam splitters separate the three channels, and a focal reducing optics provide scales of $3^{\prime \prime}$ per pixel on the $256 \times 256$ NICMOS3 arrays used for the two infrared channels, and $1^{\prime \prime}$ per pixel on the $1024 \times 1024$ Tektronix CCD detector of the $I$ channel.

The image data were processed with the standard DENIS software pipeline (Borsenberger 1997; Borsenberger et al., in preparation) at the Paris Data Analysis Center (PDAC). The thermal background produced by the instrument and the sky emission is derived from a local clipped mean along the strip. Flatfield corrections are derived from observation of the sunrise sky. Source extraction and photometry are performed at PDAC, using a space-varying kernel algorithm (Alard 2000). The astrometry of the individual DENIS frames is referenced to the USNO-A2.0 catalogue, whose $\sim 1^{\prime \prime}$ accuracy therefore determines the absolute precision of the DENIS positions.

With a $100 \%$ completeness level at $I \sim 18.0, J \sim 16.0$ and $K_{\mathrm{s}} \sim 13.0$, the DENIS survey is sensitive to M dwarfs out to large distances (Table 1), well beyond the $25 \mathrm{pc}$ limit of the CNS3 (1991). In this part of the HR diagram the $I-J$ (or alternately $I-K$ ) colour index is an excellent spectral type and luminosity estimator (Leggett 1992). The DENIS data therefore provide immediate identification of $\mathrm{M}$ dwarf candidates from $I-J>1.0$, and, under the assumption that they are indeed dwarfs, a good estimate of their distance through colour-magnitude relations. Color-magnitude relations have yet to be established for the native DENIS photometric system, and we therefore resort to a relation in the standard Cousins-CIT system to derive distances for red stars in the DENIS database. A preliminary comparison of the two photometric systems for very late $\mathrm{M}$ dwarfs shows differences of $\sim 0.1 \mathrm{mag}$ between the $K$ bands, of slightly less than 0.05 mag between the $J$ bands, and below 0.05 mag between the two 
Table 2. Position and observation information for the 30 new nearby star candidates.

\begin{tabular}{|c|c|c|c|c|c|c|}
\hline DENIS Name & Other name & $\alpha_{2000}$ & $\delta_{2000}$ & $\begin{array}{l}\text { DENIS } \\
\text { Epoch }\end{array}$ & $\begin{array}{l}\text { Number of } \\
\text { observations }\end{array}$ & $\begin{array}{l}\text { Time } \\
\text { baseline [yr] }\end{array}$ \\
\hline DENIS-P J0004575-170937 & $\ldots$ & 000457.54 & -170937.0 & 1999.521 & 4 & 47.834 \\
\hline DENIS-P J0013466-045736 & LHS 1042 & 001346.60 & -045736.5 & 1999.846 & 4 & 45.173 \\
\hline DENIS-P J0019275-362015 & $\ldots$ & 001927.53 & -362015.7 & 1999.877 & 3 & 21.064 \\
\hline DENIS-P J0041353-562112 & $\ldots$ & 004135.39 & -562112.9 & 1999.803 & 3 & 20.984 \\
\hline DENIS-P J0145434-372959 & $\ldots$ & 014543.49 & -372959.3 & 1999.877 & 3 & 22.165 \\
\hline DENIS-P J0253444-795913 & $\ldots$ & 025344.41 & -795913.6 & 1999.699 & 3 & 21.921 \\
\hline DENIS-P J0312251+002158 & $\ldots$ & 031225.11 & +002158.6 & 1999.704 & 3 & 48.020 \\
\hline DENIS-P J0324268-772705 & $\ldots$ & 032426.88 & -772705.5 & 1999.855 & 3 & 22.077 \\
\hline DENIS-P J0413398-270428 & LP 890- 2 & 041339.81 & -270428.9 & 1999.838 & 4 & 41.869 \\
\hline DENIS-P J0602542-091503 ${ }^{+}$ & LHS 1810 & 060254.26 & -091503.8 & 1999.929 & 3 & 17.040 \\
\hline DENIS-P J0848189-201911 ${ }^{+}$ & LHS 2049 & $0848 \quad 18.94$ & -201911.4 & 1999.258 & 3 & 21.014 \\
\hline DENIS-P J1003191-010507 ${ }^{+}$ & LHS 5165 & 100319.18 & -010507.7 & 1999.030 & 3 & 13.011 \\
\hline DENIS-P J1136409-075511 & LP $673-63$ & 113640.99 & -075511.7 & 1999.373 & 4 & 45.195 \\
\hline DENIS-P J1216101-112609 & LP 734-87 & 121610.16 & -112609.9 & 1999.203 & 4 & 44.948 \\
\hline DENIS-P J1223562-275746 & LHS $325 \mathrm{a}$ & 122356.27 & -275746.7 & 1999.304 & 4 & 41.162 \\
\hline DENIS-P J1236153-310646 & LP 909-55 & 123615.32 & -310646.0 & 1999.249 & 4 & 41.923 \\
\hline DENIS-P J1357149-143852 & $\ldots$ & 135714.97 & -143852.6 & 1999.225 & 4 & 44.959 \\
\hline DENIS-P J1406493-301828 ${ }^{+}$ & LHS 2859 & 140649.33 & -301828.0 & 1999.395 & 3 & 24.927 \\
\hline DENIS-P J1412069-041348 & LP $679-32$ & 141206.98 & -041348.2 & 1999.444 & 4 & 42.118 \\
\hline DENIS-P J1553251-044741 & $\ldots$ & 155325.13 & -044741.3 & 1999.581 & 4 & 45.170 \\
\hline DENIS-P J1614252-025100 & LP 624-54 & 161425.20 & -025100.5 & 1999.280 & 4 & 45.763 \\
\hline DENIS-P J2002134-542555 & $\ldots$ & 200213.42 & -542555.7 & 1999.581 & 4 & 23.077 \\
\hline DENIS-P J2049527-171608 & LP $816-10$ & 204952.72 & -171608.6 & 1999.518 & 3 & 44.946 \\
\hline DENIS-P J2107247-335733 & $\ldots$ & 210724.73 & -335733.3 & 1999.581 & 4 & 19.812 \\
\hline DENIS-P J2134222-431610 & WT 792 & 213422.27 & -431610.4 & 1999.767 & 3 & 21.189 \\
\hline DENIS-P J2202112-110945* & LP 759-17 & 220211.28 & -110945.8 & 1999.419 & 4 & 45.672 \\
\hline DENIS-P J2213504-634210 & WT 887 & 221350.47 & -634210.0 & 1999.537 & 3 & 22.976 \\
\hline DENIS-P J2331217-274949 & $\ldots$ & 233121.73 & -274949.9 & 1999.869 & 5 & 45.119 \\
\hline DENIS-P J2333405-213353 & LHS 3970 & 233340.59 & -213353.2 & 1999.770 & 4 & 45.176 \\
\hline DENIS-P J2353594-083331 & $\ldots$ & 235359.44 & -083331.6 & 1999.693 & 3 & 46.077 \\
\hline
\end{tabular}

+ Objects selected from LHS initially.

* Already listed by Gizis et al. (2000).

Columns 1 \& 2: object name in the DENIS data base and other identification if available.

Columns 3, 4 \& 5: DENIS Position with respect to equinox J2000 at DENIS epoch.

Columns 6 \& 7: number of position observations and time baseline.

$I$ bands (Delfosse 1997b). We therefore choose to determine distances from the $\left(I-J, M_{I}\right)$ relation, which is least affected by the neglected colour terms, and note that systematic errors from adopting the Cousins-CIT relation are much smaller than the intrinsic dispersion of that relation. The two analytic $\left(I-J, M_{I}\right)$ relations in the literature, from Leggett (1992) and Tinney (1993), respectively concentrate on early and late $\mathrm{M}$ dwarfs. For consistency across the $\mathrm{M}$ spectral class (and for convenience), we therefore fitted a polynomial relation to the photometric and trigonometric data of Leggett (1992), Tinney (1993) and Tinney et al. (1995), adding some photometry from Alonso et al. (1996) and Weis (1993). Figure 1 shows the resulting relation,

$M_{I}=4.97-4.76(I-J)+10.03(I-J)^{2}-4.07(I-J)^{3}$ $+0.53(I-J)^{4}$, valid for $1.0<I-J<3.0$ and demonstrates its fair agreement with the theoretical track of Baraffe et al. (1998). The new polynomial fit complements the $\left(I-K, M_{K}\right)$ relation derived by Tinney et al. (1995) for the same spectral type range.

With the above Color-Magnitude relation as tool, we have searched the first 2110 squares degrees processed by PDAC (Fig. 2) for new members of the solar neighbourhood. We select all DENIS sources with $\left|b_{\mathrm{II}}\right| \geq 30^{\circ}$ that have the $I-J$ colour of an M dwarf $(1.0 \leq I-J \leq 3.0$; Leggett 1992). We then compute their photometric distance $\left(D_{\text {phot }}\right)$ from the DENIS photometry and the above polynomial colour-magnitude relations, and retain those with $D_{\text {phot }} \leq 30 \mathrm{pc}$. In the present paper we focus on the $2.0<I-J<3.0$ range, or spectral types of approximately M 6 to M 8. Bluer nearby M dwarfs are brighter and hence less likely to have been overlooked. At present we therefore give them a lower priority. Redder ones, like 
Table 3. 34 red DENIS stars with $\mu<0.1 \mathrm{yr}^{-1}$, not kept for further investigation in the present paper.

\begin{tabular}{|c|c|c|c|c|c|c|}
\hline DENIS Name & $\alpha_{2000}$ & $\delta_{2000}$ & $\begin{array}{l}\text { DENIS } \\
\text { Epoch }\end{array}$ & $I$ & $I-J$ & $J-K$ \\
\hline DENIS-P J0013093-002551 & 001309.34 & -002551.5 & 1999.838 & 14.37 & 2.22 & 0.88 \\
\hline DENIS-P J0100021-615627 & $\begin{array}{lll}01 & 00 & 02.13\end{array}$ & -615627.1 & 1999.964 & 15.01 & 2.42 & 0.94 \\
\hline DENIS-P J0250072-860930 & 025007.20 & -860930.0 & 1999.712 & 9.26 & 2.09 & 1.41 \\
\hline DENIS-P J0441247-271453 & 044124.70 & -271453.6 & 1999.063 & 8.92 & 2.19 & 1.20 \\
\hline DENIS-P J1117420-264453 & 111742.08 & -264453.8 & 1999.266 & 11.71 & 2.16 & 0.56 \\
\hline DENIS-P J1236396-172216 & 123639.61 & -172216.9 & 1999.384 & 13.91 & 2.14 & 1.14 \\
\hline DENIS-P J1400335-271656 & 140033.51 & -271656.2 & 1999.348 & 9.69 & 2.09 & 1.26 \\
\hline DENIS-P J1405376-221515 & 140537.64 & -221515.0 & 1999.285 & 9.49 & 2.09 & 1.29 \\
\hline DENIS-P J1427297-264040 & 142729.71 & -264040.8 & 1999.419 & 9.68 & 2.12 & 1.20 \\
\hline DENIS-P J1510397-212524 & 151039.72 & -212524.9 & 1999.384 & 10.06 & 2.22 & 1.18 \\
\hline DENIS-P J1525014-032359 & 152501.46 & -032359.5 & 1999.351 & 9.25 & 2.09 & 1.08 \\
\hline DENIS-P J1552237-033520 & 155223.78 & -033520.7 & 1999.534 & 12.02 & 2.07 & 1.37 \\
\hline DENIS-P J1552551-045215 & 155255.19 & -045215.3 & 1999.534 & 10.21 & 2.01 & 1.38 \\
\hline DENIS-P J1553186-025919 & 155318.65 & -02 5919.3 & 1999.581 & 13.12 & 2.04 & 1.36 \\
\hline DENIS-P J1615446-040526 & 161544.69 & -040526.2 & 1999.353 & 9.67 & 2.03 & 1.19 \\
\hline DENIS-P J1618120-044221 & 161812.09 & -044221.8 & 1999.386 & 11.32 & 2.26 & 1.28 \\
\hline DENIS-P J2024329-294402 & 202432.96 & -294402.6 & 1999.392 & 10.45 & 2.13 & 1.26 \\
\hline DENIS-P J2032270-273058 & 203227.03 & -273058.4 & 1999.534 & 10.76 & 2.45 & 1.18 \\
\hline DENIS-P J2034203-263652 & 203420.33 & -263652.2 & 1999.712 & 11.27 & 2.23 & 1.12 \\
\hline DENIS-P J2044066-173457 & 204406.68 & -173457.3 & 1999.606 & 11.28 & 2.42 & 1.29 \\
\hline DENIS-P J2055240-322600 & 205524.07 & -322600.8 & 1999.669 & 10.73 & 2.10 & 1.30 \\
\hline DENIS-P J2056329-782540 & 205632.90 & -782540.1 & 1999.660 & 10.43 & 2.08 & 1.20 \\
\hline DENIS-P J2058075-730350 & 205807.55 & -730350.4 & 1999.660 & 11.89 & 2.35 & 1.29 \\
\hline DENIS-P J2103375-783831 & 210337.56 & -783831.5 & 1999.658 & 11.42 & 2.08 & 1.30 \\
\hline DENIS-P J2124575-341655 & 212457.51 & -341655.9 & 1999.559 & 13.60 & 2.37 & 1.32 \\
\hline DENIS-P J2125399-100526 & 212539.98 & -100526.1 & 1999.482 & 12.25 & 2.10 & 0.59 \\
\hline DENIS-P J2130021-815158 & 213002.15 & -815158.6 & 1999.510 & 10.33 & 2.17 & 1.37 \\
\hline DENIS-P J2155040-165535 & 215504.06 & -165535.1 & 1999.712 & 12.46 & 2.37 & 0.40 \\
\hline DENIS-P J2203522-593300 & 220352.29 & -593300.7 & 1999.649 & 11.29 & 2.43 & 1.16 \\
\hline DENIS-P J2206227-204706 & 220622.78 & -204706.0 & 1999.611 & 15.09 & 2.67 & 1.22 \\
\hline DENIS-P J2225004-121606 & 222500.48 & -121606.9 & 1999.447 & 10.38 & 2.25 & 1.19 \\
\hline DENIS-P J2226443-750342 & 222644.36 & -750342.7 & 1999.814 & 15.20 & 2.84 & 1.20 \\
\hline DENIS-P J2312219-091513 & 231221.98 & -091513.5 & 1999.482 & 13.70 & 2.99 & 0.29 \\
\hline DENIS-P J2334544-193232 & 233454.49 & -193232.4 & 1999.707 & 14.63 & 2.94 & 0.54 \\
\hline
\end{tabular}

Column 1: object name.

Columns 2, 3 \& 4: DENIS Position with respect to equinox J2000 at DENIS epoch.

Columns 5, 6 \& 7: DENIS I-magnitude and colours.

DENIS-P J104814.7-395606.1 (Delfosse et al. 2001), are addressed by the DENIS Brown Dwarf program (Delfosse et al., in preparation).

These criteria are met by 60 objects (Tables 2, 3), 13 of which are listed in proper motion catalogues but had no distance estimate (except WT 792, discussed below). At that stage the sample is a mix of distant giants and nearby dwarfs, which cannot be completely separated using the DENIS photometry alone: the dwarf and giant sequences are distinct in the DENIS $I-J / J-K$ colour-colour diagram, but not sufficiently separated to distinguish the two classes with $100 \%$ completeness and reliability.

\subsection{Additional candidates from the LHS catalogue}

We additionally browsed the LHS catalogue (Luyten 1979) for objects that match the colour and photometric distance criteria, but which had been missed because of our galactic latitude cutoff, or whose DENIS observations had not yet been entered in the database. To narrow down that search, we looked for unambiguous DENIS identifications to southern LHS stars matching $B_{\text {Luyten }}>16.0$, $R_{\text {Luyten }}>15.0$, and $B_{\text {Luyten }}-R_{\text {Luyten }}>1.5$, and then applied the colour and photometric distance cutoffs. This identified 4 additional candidates: LHS 1810, 2049, 2859 and 5165, which are added in the Tables.

\section{Proper motions and $B, R$ photometry}

As an initial step towards rejecting giants, we determine the proper motion of the candidates from a comparison of the DENIS position with archival Schmidt plates digitized on the MAMA microdensitometer. Objects fainter than $R=10$ with a proper motion $\mu \geq 0.1 \mathrm{yr}^{-1}$ must 
Table 4. Proper motion of the 30 Nearby Star candidates.

\begin{tabular}{lllll}
\hline \hline DENIS Name & $\begin{array}{l}\mu_{\alpha} \\
{\left[\mathrm{yr}^{-1}\right]}\end{array}$ & $\begin{array}{l}\mu_{\delta} \\
{\left[\mathrm{yr}^{-1}\right]}\end{array}$ & $\begin{array}{l}\mu_{\text {total }} \\
{\left[\mathrm{yr}^{-1}\right]}\end{array}$ & $\begin{array}{l}\mu_{\mathrm{L} / \mathrm{W}} \\
{\left[\mathrm{yr}^{-1}\right]}\end{array}$ \\
\hline J0004575-170937* & +0.146 & -0.011 & 0.146 & $\ldots$ \\
J0013466-045736 & +0.584 & -0.153 & 0.604 & 0.619 \\
J0019275-362015* & +0.152 & -0.097 & 0.180 & $\ldots$ \\
J0041353-562112* & +0.121 & -0.064 & 0.137 & $\ldots$ \\
J0145434-372959* & +0.424 & -0.118 & 0.440 & $\ldots$ \\
J0253444-795913* & +0.074 & +0.081 & 0.110 & $\ldots$ \\
J0312251+002158* & +0.173 & -0.028 & 0.176 & $\ldots$ \\
J0324268-772705* & +0.287 & +0.184 & 0.341 & $\ldots$ \\
J0413398-270428 & +0.234 & -0.023 & 0.235 & 0.230 \\
J0602542-091503 & +0.172 & -0.616 & 0.639 & 0.608 \\
J0848189-201911 & +0.342 & -0.593 & 0.685 & 0.633 \\
J1003191-010507 & -0.497 & +0.056 & 0.500 & 0.491 \\
J1136409-075511 & -0.171 & +0.144 & 0.224 & 0.223 \\
J1216101-112609 & +0.041 & -0.219 & 0.223 & 0.212 \\
J1223562-275746 & -1.239 & +0.323 & 1.280 & 1.293 \\
J1236153-310646 & +0.161 & -0.083 & 0.181 & 0.191 \\
J1357149-143852* & -0.355 & +0.023 & 0.356 & $\ldots$ \\
J1406493-301828 & -0.855 & -0.059 & 0.857 & 0.814 \\
J1412069-041348 & +0.292 & -0.152 & 0.329 & 0.342 \\
J1553251-044741* & -0.034 & -0.121 & 0.126 & $\ldots$ \\
J1614252-025100 & +0.003 & +0.367 & 0.367 & 0.401 \\
J2002134-542555* & +0.060 & -0.364 & 0.369 & $\ldots$ \\
J2049527-171608 & +0.303 & -0.102 & 0.320 & 0.305 \\
J2107247-335733* & +0.344 & -0.367 & 0.503 & $\ldots$ \\
J2134222-431610 & +0.142 & -0.792 & 0.804 & 0.785 \\
J2202112-110945 & +0.137 & -0.182 & 0.228 & 0.210 \\
J2213504-634210 & +0.140 & +0.166 & 0.217 & 0.195 \\
J2331217-274949* & +0.087 & +0.738 & 0.744 & $\ldots$ \\
J2333405-213353 & +0.682 & -0.336 & 0.761 & 0.794 \\
J2353594-083331* & -0.039 & -0.367 & 0.369 & $\ldots$ \\
\hline & & & & \\
& & & & \\
& & & $\ldots$ \\
& &
\end{tabular}

* Not previously known as a high proper motion star.

Columns 2, $3 \& 4: \mu_{\alpha}, \mu_{\delta}, \mu_{\text {total }}$ our measurement, in $\operatorname{arcsec} \mathrm{yr}^{-1}$.

Column 5: literature total proper motion, from Luyten (1979, 1980) or Wroblewski et al. (1994) when available.

be dwarfs: a red giant with such an apparent magnitude and proper motion would have a very large and improbable space velocity $\left(\geq 1000 \mathrm{~km} \mathrm{~s}^{-1}\right)$, much larger than the Galactic escape speed (Meillon 1999). Conversely, some small fraction of the nearby dwarfs must have proper motions below our cutoff. To estimate that proportion, we counted the fraction of the stars within $25 \mathrm{pc}$ in both the CNS3 and the Hipparcos catalogue that have a proper motion smaller than $0.1 \mathrm{yr}^{-1}$. In the CNS3, this fraction of "slow" stars is about $13 \%$ for limiting magnitudes of $V<7,8$ and 9. In the Hipparcos catalogue and for the same limiting magnitudes it is much lower, only $6.5 \%$. The CNS3 is considered complete to $V=9$. The Hipparcos catalogue is complete up to $V=7.3$, and nearly complete up to at least 9 for nearby stars, as potential nearby stars were systematically included in the Hipparcos Input catalogue. Incompleteness therefore cannot explain the discrepancy between the fractions of low proper-motion stars

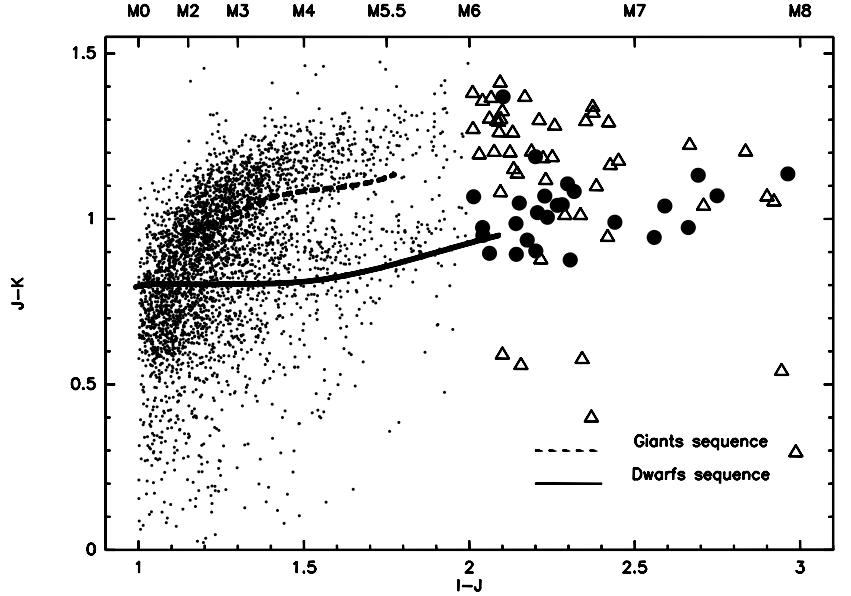

Fig. 2. All DENIS sources with the $I-J$ colour of an M-dwarf $(1.0<I-J<3.0)$ detected in the first 2110 squares degrees processed by PDAC, with a photometric distance $\leq 30 \mathrm{pc}$ and a galactic latitude $\geq 30^{\circ}$. The dots represent the early Mstars $(1.0<I-J<3.0)$. Amongst the 60 DENIS sources in the 2.0-3.0 range (corresponding to a late-M spectral class) the solid circles represent the objects with a proper motion $\mu>0.1 \mathrm{yr}^{-1}$, and the triangles those with a smaller proper motion. The solid and dashed lines respectively represent the dwarf and giant sequences of Bessell \& Brett (1988), for slightly different filters. The (indicative) spectral type labels on the top axis are adopted from Leggett (1992).

in the two catalogues. A careful comparison between the two lists of "slow" stars shows that half of the slow CNS3 objects have a Hipparcos distance larger than 25 pc, and should therefore not be taken into consideration. The fraction of nearby stars lost due to our proper motion cut-off is therefore of the order of $6 \%$. Our future goal is to obtain spectra of all candidates, independently of their proper motions, to assemble a complete and unbiased inventory of the solar neighbourhood for the high galactic latitude southern sky.

To obtain $B$ and $R$ photometry of the candidates and determine their proper motions, we identified all Survey plates in the CAI (http://dsmama.obspm.fr/) plate vault that contained images of the nearby star candidates. Depending on the declination of the candidates, the following plates were available: POSS $I$ for $-30^{\circ}<\delta<0^{\circ}$, SRC- $J$ for $-90^{\circ}<\delta<0^{\circ}$, SRC- $R$ for $-17^{\circ}<\delta<0^{\circ}$, and ESO- $R$ for $\delta<-17^{\circ}$. We used the MAMA microdensitometer (Berger et al. 1991) at CAI to digitize the survey plates, and analysed the resulting images with SExtractor (Bertin \& Arnouts 1996). The SExtractor source parameters were calibrated using the GSPC-2 (Postman et al. 1997; Bucciarelli et al. 2001) and ACT catalogues (Urban et al. 1998) as photometric and astrometric references to produce $B$ and $R$ magnitudes (Table 5), and equatorial coordinates at the epoch of the plate. Absolute proper motions were then determined through a least square fit to the positions at the 2 to 4 Schmidt plate epochs and the DENIS survey epoch. The proper motion errors range between 8 and 19 mas/year, depending on the object 

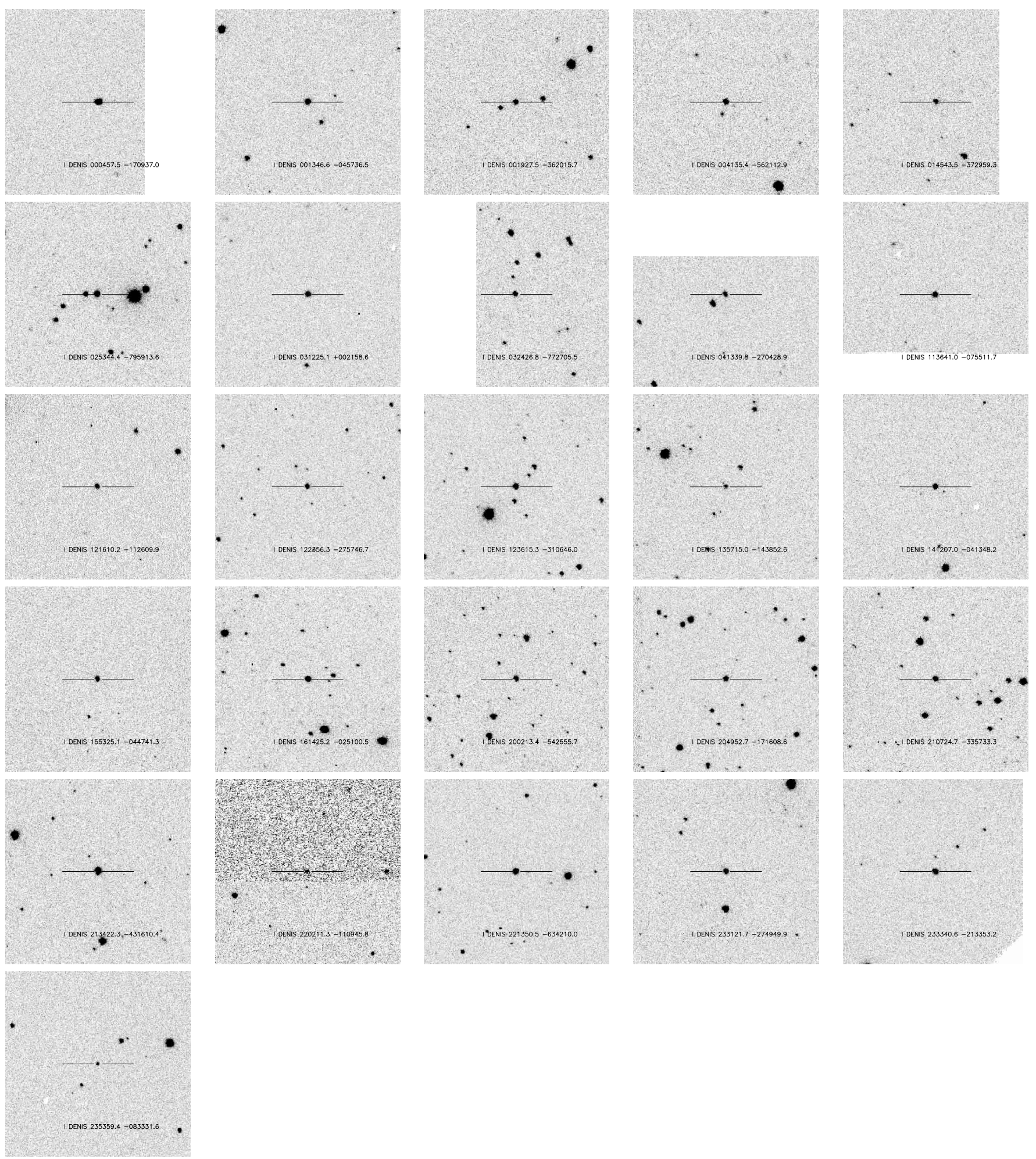

Fig. 3. $I$-band finding charts for the objects listed in Table 2 . The size of each chart is $\sim 3.5^{\prime} \times 3.5^{\prime}$. North is up and east is to the left. The 4 stars selected directly from the LHS are not included.

magnitude and the available time baseline. The magnitude errors are $\pm 0.3 \mathrm{mag}$ for $B$ and $\pm 0.2 \mathrm{mag}$ for $R$. From the full sample of 60 objects, 34 have no proper motion above the $0.1 \mathrm{yr}^{-1}$ level and are listed in Table 3 . They are not studied in this paper, and leave us with 26 high quality nearby star candidates (Table 2) to which the 4 LHS stars are added (finding charts in Fig. 3). Of the 30 propermotion objects, 13 were previously unknown. 2 of those have a proper motion, $\mu>0.5 \mathrm{yr}^{-1}$, that would have qualified them for inclusion in the LHS. An additional 5 have $\mu>0.2 \mathrm{yr}^{-1}$ and could have been included in the NLTT. The 17 remaining objects belong to the LHS, the NLTT, or 
Table 5. MAMA and DENIS photometry, and estimated distances, for the 30 DENIS red dwarf candidates.

\begin{tabular}{|c|c|c|c|c|c|c|c|c|c|c|}
\hline DENIS objects & $B$ & $R$ & $I$ & $I-J$ & $J-K$ & $M_{I}$ & $M_{K}$ & $\begin{array}{l}\operatorname{Dist}_{I} \\
{[\mathrm{pc}]}\end{array}$ & $\begin{array}{l}\operatorname{Dist}_{K} \\
{[\mathrm{pc}]} \\
\end{array}$ & $\begin{array}{l}V_{\mathrm{t}} \\
{\left[\mathrm{km} \mathrm{s}^{-1}\right]}\end{array}$ \\
\hline J0004575-170937 & 18.2 & 15.6 & 13.00 & 2.03 & 0.93 & 11.59 & 8.78 & 19.1 & 17.8 & 13.2 \\
\hline J0013466-045736 & 19.5 & 16.8 & 13.88 & 2.44 & 0.99 & 12.73 & 9.69 & 17.0 & 14.2 & 48.7 \\
\hline J0019275-362015 & 18.2 & 17.0 & 14.30 & 2.14 & 0.89 & 11.95 & 8.95 & 29.6 & 29.1 & 25.3 \\
\hline J0041353-562112 & 20.6 & 18.7 & 14.68 & 2.74 & 1.07 & 13.37 & 10.12 & 18.2 & 14.1 & 11.8 \\
\hline J0145434-372959 & 20.2 & 17.7 & 15.05 & 2.56 & 0.94 & 13.00 & 9.79 & 25.7 & 22.5 & 53.6 \\
\hline J0253444-795913 & 18.7 & 16.2 & 13.47 & 2.15 & 0.98 & 11.98 & 9.17 & 19.9 & 17.1 & 10.4 \\
\hline $\mathrm{J} 0312251+002158$ & 20.4 & 17.0 & 14.30 & 2.17 & 0.93 & 12.03 & 9.11 & 28.4 & 26.2 & 23.7 \\
\hline J0324268-772705 & 20.4 & 17.3 & 14.36 & 2.26 & 1.04 & 12.29 & 9.49 & 26.0 & 20.6 & 42.0 \\
\hline J0413398-270428 & 22.0 & 17.7 & 14.45 & 2.26 & 0.99 & 12.29 & 9.40 & 27.1 & 22.9 & 30.2 \\
\hline J0602542-091503 & 17.3 & 15.6 & 12.98 & 2.12 & 0.85 & 11.88 & 8.81 & 16.6 & 17.4 & 48.9 \\
\hline J0848189-201911 & 19.7 & 17.2 & 14.51 & 2.21 & 0.98 & 12.15 & 9.29 & 29.7 & 25.4 & 88.3 \\
\hline J1003191-010507 & 21.0 & 18.0 & 14.94 & 2.62 & 1.08 & 13.12 & 10.02 & 23.1 & 17.6 & 51.7 \\
\hline J1136409-075511 & 17.8 & 16.5 & 14.31 & 2.20 & 0.90 & 12.12 & 9.11 & 27.4 & 26.3 & 29.1 \\
\hline J1216101-112609 & 19.5 & 17.1 & 14.72 & 2.30 & 1.10 & 12.39 & 9.65 & 29.2 & 21.6 & 30.9 \\
\hline $\mathrm{J} 1223562-275746$ & 19.6 & 16.4 & 14.19 & 2.30 & 0.88 & 12.39 & 9.27 & 22.9 & 22.3 & 138.9 \\
\hline J1236153-310646 & 18.5 & 16.7 & 13.97 & 2.23 & 1.01 & 12.21 & 9.39 & 22.5 & 18.6 & 19.3 \\
\hline J1357149-143852 & 21.1 & 18.5 & 15.55 & 2.69 & 1.14 & 13.27 & 10.13 & 28.5 & 20.8 & 48.1 \\
\hline J1406493-301828 & 18.5 & 15.7 & 13.42 & 2.13 & 1.01 & 11.91 & 9.19 & 20.0 & 16.5 & 77.5 \\
\hline J1412069-041348 & 19.1 & 16.3 & 13.66 & 2.04 & 0.98 & 11.63 & 8.93 & 25.5 & 22.0 & 39.8 \\
\hline J1553251-044741 & 17.4 & 15.1 & 13.67 & 2.10 & 1.37 & 11.82 & 9.75 & 23.4 & 12.3 & 14.0 \\
\hline $\mathrm{J} 1614252-025100$ & 19.3 & 17.3 & 13.63 & 2.29 & 1.04 & 12.37 & 9.54 & 17.9 & 14.2 & 31.1 \\
\hline J2002134-542555 & 19.4 & 17.1 & 13.89 & 2.20 & 1.18 & 12.12 & 9.62 & 22.6 & 15.1 & 39.5 \\
\hline J2049527-171608 & 18.9 & 16.8 & 14.16 & 2.32 & 1.08 & 12.44 & 9.65 & 22.0 & 16.7 & 33.4 \\
\hline J2107247-335733 & 19.9 & 16.7 & 14.36 & 2.20 & 1.02 & 12.12 & 9.35 & 28.0 & 22.8 & 66.8 \\
\hline $\mathrm{J} 2134222-431610$ & 17.6 & 15.3 & 12.78 & 2.02 & 1.06 & 11.56 & 9.07 & 17.5 & 13.4 & 66.7 \\
\hline J2202112-110945 & 20.7 & 17.9 & 15.11 & 2.66 & 0.98 & 13.21 & 9.96 & 24.0 & 20.1 & 25.9 \\
\hline J2213504-634210 & 18.7 & 16.3 & 13.05 & 2.16 & 1.04 & 12.01 & 9.31 & 16.2 & 12.8 & 16.7 \\
\hline $\mathrm{J} 2331217-274949$ & 20.4 & 17.7 & 14.25 & 2.59 & 1.04 & 13.06 & 9.94 & 17.3 & 13.6 & 61.0 \\
\hline J2333405-213353 & 19.3 & 16.5 & 13.89 & 2.06 & 0.90 & 11.69 & 8.78 & 27.5 & 26.9 & 98.2 \\
\hline J2353594-083331 & $* *$ & 18.6 & 15.93 & 2.97 & 1.13 & 13.92 & 10.32 & 25.3 & 20.0 & 44.3 \\
\hline
\end{tabular}

** Too faint for the plate.

Column 1: object name. Columns 2, 3: $B, R$ magnitudes determined from the plates using GSPC and GSPC-2 calibrations. Columns 4, 5, 6: DENIS $I$-magnitude and colours. Column 7: $M_{I}$ absolute $I$ band magnitude, from the colour magnitude relation derived in the present paper. Column 8: $M_{K}$ absolute $K$ band magnitude, from the Tinney et al. (1995) relation. Columns 9, 10: Dist $_{I}$, Dist ${ }_{K}$ distances estimated from $M_{I}$ and $M_{K}$ respectively. Column 11: $V_{\mathrm{t}}$ tangential velocity, computed from $\operatorname{Dist}_{I}$ and the proper motion.

the Catalogue of New proper motion stars (Wroblewski \& Torres 1994). For those, the difference between our proper motions and the literature values has a mean of 9 mas $\mathrm{yr}^{-1}$

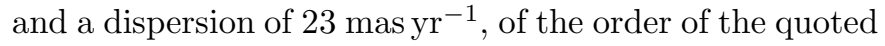
LHS accuracy. Table 4 lists our proper motion measurements, and the literature proper motion when available. Figure 2, which distinguishes the high and low proper motion stars in the $I-J / J-K$ diagram, immediately shows that the high proper motion stars belong to the dwarf sequence, with only one exception, DENIS-P J1553251044741. On the other hand, some low proper motion stars do, as expected, seem to belong to the dwarf sequence.

\section{Discussion}

In addition to the $(I, I-J)$ distances computed from the relation derived above, Table 5 contains $(I, I-K)$ distances derived using the $\left(I-K, M_{K}\right)$ colour-magnitude relation of Tinney et al. (1995) for $1.2<I-K<5.4$. The latter are systematically somewhat smaller, most likely because of our use of DENIS $K_{\mathrm{s}}$ magnitudes and a colourmagnitude relation established in the Cousins-CIT photometric system. The $(I, I-J)$ distances are therefore most likely more reliable. Carpenter (2001) carefully derived relations for colour transformations between 2MASS and other systems, including CIT and DENIS. However the DENIS data he used is the part publicly released at CDS and reduced by the Leiden Data Center (LDAC), and covers only a very small fraction of the DENIS data. The data presented here was reduced by the Paris Data Center (PDAC) which uses slightly different methods and relations. Therefore the Carpenter's equations should not be used here. We note that Patterson et al. (1998) estimate a distance of 22 pc to WT 792 from $V R I$ photometry

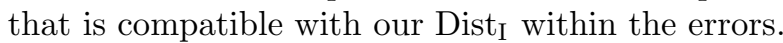


It must be realized that the distances in Table 5 carry considerable uncertainties, as any photometric parallaxes do. The intrinsic scatter of the colour-luminosity relation (Fig. 1) is $\pm 1 \mathrm{mag}$, which on a star by star basis translates into a $45 \%$ random distance error. At a lower level, the poynomial relations most likely have systematic errors at some colours: 0.3 magnitude local errors (i.e. $14 \%$ distance errors) can quite easily creep in when fitting a phenomenological model to such a dispersed diagram.

Finally, some of the 30 objects must be multiple systems, whose distances are underestimated by attributing their luminosity to one single star.

Tangential velocities calculated from the proper motions and distances, are listed in the last column of Table 5; 4 stars have tangential velocities larger than $70 \mathrm{~km} \mathrm{~s}^{-1}$ : LHS 2049; 325a; 2859; 3970. Of those LHS 325a and LHS 2049 were previously recognized as late-M stars with high reduced proper motion $(H=m+5 \log \mu+5)$ by Bessell (1991). LHS 325a is photometrically classified as an old-disk star by Leggett (1992).

The sample of low proper motion objects (Table 3) probably contains a few nearby stars, that happen to have a low tangential velocity, and is presently under study.

The $I-J$ and $I-K$ colours of the 30 nearby star candidates indicate spectral types of M 6 to M 8, which we plan to ascertain through low-resolution spectroscopy. Such objects have masses of $0.1 M_{\odot}$ (Delfosse et al. 2000) or lower.

The 2110 square degrees explored here contain 26 stars with $2.0<I-J<3.0, \mu>0.1 \mathrm{yr}^{-1}$, and a photometric distance $d<30 \mathrm{pc}$. The whole celestial sphere therefore contains $\sim 500$ similar objects, of which $\sim 60 \%$, or 300 , will have a photometric distance below 25 pc. Malmquist bias from our approximate photometric distances must bias this number up, and the number of stars that are actually within 25 pc must be significantly smaller, perhaps by as much as a factor of 2 . The $(R-I)_{\mathrm{C}}$ colour index that corresponds to our $I-J=2.0$ cutoff is $(R-I)_{\mathrm{C}}=2.0$ (Leggett 1992), or about 1.6 in the Kron system used in the CNS3. The whole CNS3 contains only 23 stars with $(R-I)_{\text {Kron }}>1.6$. An effort similar to ours over the whole celestial sphere would thus identify 150 to 250 new stars within 25 pc.

\section{Conclusion}

Many new nearby stars have been recently identified thanks to the near-IR sky surveys: DENIS and 2MASS have proved to be valuable resources for the identification of new nearby stars. In the present paper, we identify 60 photometric nearby star candidates in 2110 square degrees, with distances that lie between 15 and 30 pc. 26 of those have large proper motions that exclude that they are distant giants, 13 of which are new; 4 additional known LHS stars were recognized as nearby star candidates outside the main search area. A few stars have large tangential velocities and may be subdwarfs, whose distances would then be slightly overestimated. We plan to obtain lowresolution spectra for all candidates in the near future.

Acknowledgements. We are grateful to the DENIS consortium for providing the DENIS data, and to René Chesnel for scanning and pre-reducing the photographic plates. The long-term loan of POSS I plates by the Leiden Observatory to Observatoire de Paris is gratefully acknowledged.

We thank the referee, Dr. Hartmut Jahreiß, for his prompt and very constructive report.

\section{References}

Alard, C. 2000, A\&AS, 144, 363

Allard, F., Hauschildt, P. H., Baraffe, I., \& Chabrier, G. 1996, ApJ, 465, L123

Alonso, A., Arribas, S., \& Martinez-Roger, C. 1994, A\&AS, 107,365

Baraffe, I., Chabrier, G., Allard, F., \& Hauschildt, P. H. 1995, ApJ, 446, L35

Baraffe, I., Chabrier, G., Allard, F., \& Hauschildt, P. H. 1998, A\&A, 337, 403

Berger, J., \& Cordoni, J. -P., Fringant, A.-M., et al. 1991, A\&AS, 87, 389

Bertin, E., \& Arnouts, S. 1996, A\&AS, 117, 393

Bessell, M. S., \& Brett, J. M. 1988, PASP, 100, 1134

Bessell, M. S. 1991, AJ, 101, 662

Borsenberger, J. 1997, in the 3rd DENIS Euroconference, The impact of large scale near-infrared surveys, ed. F. Garzon et al. (Kluwer Dordrecht), 121

Bucciarelli, B., Garcia Yus, J., Casalegno, R., et al. 2001, A\&A, 368,335

Burrows, A., Hubbard, W. B., Saumon, D., \& Lunine, J. I. 1993, ApJ, 406, 158

Carpenter, J. M. 2001, AJ, 121, 2851

Chabrier, G., \& Baraffe, I. 1997, A\&A, 327, 1039

D'Antona, F., \& Mazzitelli, I. 1994, ApJS, 90, 467

Delfosse, X., Tinney, C. G., Forveille, T., et al. 1997a, A\&A, 327, L25

Delfosse, X. 1997b, Ph.D. Thesis, page 43 and Fig. 2.9, Grenoble University

Delfosse, X., Forveille, T., Ségransan, D., et al. 2000, A\&A, 364,217

Delfosse, X., Forveille, T., Martin, E. L., et al. 2001, A\&A, 366, L13

Epchtein, N. 1997, in the 2nd DENIS Euroconference, The impact of large scale near-infrared surveys, ed. F. Garzon et al. (Kluwer Dordrecht), 15

Gizis, J. E., \& Reid, I. N. 1997, PASP, 109, 849

Gizis, J. E., Monet, D. G., Reid, I. N., et al. 2000, AJ, 120, 1085

Giclas, H. L., Burnham, R. Jr., \& Thomas, N. G. 1971, Lowell Northern Hemisphere Catalogue, The G Numbered Stars, Lowell Obs.

Giclas, H. L., Burnham, R. Jr., \& Thomas, N. G. 1978, Lowell Southern Hemisphere Catalogue, Lowell Obs. Bull., 164, 89

Gliese, W. 1956, ZA, 39, 1

Gliese, W., \& Jahreiß, H. 1979, A\&AS, 38, 423

Gliese, W., Jahreiß, H., \& Upgren, A.R. 1986, in The galaxy and the solar system, ed. R. Schmoluchowski et al. (The University of Arizona press), 13

Gliese, W., \& Jahreiß, H. 1991, Preliminary Version of the Third Catalogue of Nearby Stars, as available at CDS Strasbourg 
Henry, T. J., Ianna, P. A., Kirkpatrick, J. D., \& Jahreiß, H. 1997, AJ, 114, 388

Jahreiß, H., Scholz, R. D., Meusinger, H., \& Lehmann, I. 2001, A\&A, 370, 967

Leggett, S. K. 1992, ApJS, 82, 531

Luyten, W. J. 1955, A Catalogue of 1849 stars with proper motions exceeding $0^{\prime \prime} 5$ annually (LFT) (Lund Press, Minneapolis, Minn.)

Luyten, W. J. 1957, A Catalogue of 9867 stars in the southern hemisphere with proper motions exceeding 0.2 annually (LTT) (Lund Press, Minneapolis, Minn.)

Luyten, W. J. 1961, A Catalogue of 7127 stars in the northern hemisphere with proper motions exceeding 0 .' 2 annually (LTT) (Lund Press, Minneapolis, Minn.)

Luyten, W. J. 1979, Catalogue of stars with proper motions exceeding 0.5 annually (LHS) (Minneapolis, University of Minnesota)

Luyten, W. J. 1980, New Luyten catalog of stars with proper motions larger than Two Tenths of an arcsecond (NLTT) (Minneapolis, University of Minnesota)
Martin, E. L., Basri, G., Delfosse, X., \& Forveille, T. 1997, A\&A, 327, L29

Meillon, L. 1999, Ap\&SS, 265, 179

Patterson, R. J., Ianna, P. A., \& Begam, M. C. 1998, AJ, 115, 1648

Porter, J. G., Yowell, E. J., \& Smith, E. S. 1918, Publ. Cincinnati Obs., 18, No. 4

Porter, J. G., Yowell, E. J., \& Smith, E. S. 1930, Publ. Cincinnati Obs., 20, 1

Postman, M., Bucciarelli, B., Sturch C., et al. 1997, IAU Symp., 179, 379

Scholz, R. D., Meusinger, H., Jahreiß, H. 2001, A\&A, 374, L12

Tinney, C. G 1993, AJ, 105, 1169

Tinney, C. G, Reid, I. N., Gizis, J., \& Mould, J. R. 1995, AJ, 110,3014

Urban, S. E., Corbin, T. E., \& Wycoff, G. L. 1998, AJ, 115, 2161

Weis, E. W. 1993, AJ, 105, 1962

Wroblewski, H., \& Torres, C. 1994, A\&AS, 105, 179 